Review

\title{
Role of Micrornas on Immune System Components in Physiological and Pathophysiological Gut Micro Milieu
}

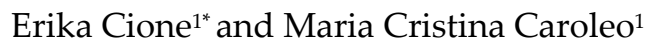 \\ 'Department of Pharmacy, Health and Nutritional Sciences "Dipartimento di Eccellenza 2018-2022" \\ University of Calabria, Edificio Polifunzionale, 87036 Rende, Italy
}

\author{
*Correspondence to: \\ Erika Cione PharmD, PhD \\ Department of Pharmacy and Health and Nutritional Sciences, University of Calabria-Italy \\ Via A. Savinio - 87036 Rende (CS). Phone 0984493193; email: erika.cione@unical.it
}

\begin{abstract}
The tissue micro environment or milieu consists of a highly dynamic population of cellular and non-cellular components which constitute a complex regulatory network aimed at maintaining the organ homeostasis. In the modern medicine the discovery of miRNAs is undoubtedly a promising field of research and they are essential in orchestrating immune system logic and their release in the gut micro milieu can directly affect bacterial gene expression. Here, we briefly review the role of microRNAs, focuses on their role on immune system components in physiological and pathophysiological gut micro milieu.
\end{abstract}

Key words: Gut micro milieu, immunity and microRNAs

\section{Introduction}

The tissue micro environment or milieu consists of a highly dynamic population of cellular and non-cellular components which constitute a complex regulatory network aimed to maintain organ homeostasis. Within a given tissue, the micro milieu components, such as growth factors, different cell phenotypes and insoluble glycoproteins of the extracellular matrix, cooperate to provide both, biochemical signals and structural constraints $[1 ; 2 ; 3]$. The micro milieu components determine the appropriate cellular behavior in the tissue. In addition, they deeply affect the intracellular gene expression patterns, that in turn induce essential 
alterations in the composition of milieu itself. This latter mechanism, originally described as "dynamic reciprocity" [1] is now widely recognized to be associated with the regulation of cellular differentiation, proliferation and survival $[4 ; 5]$. Therefore, in order to preserve and maintain the physiological commitment cells residing within a given tissue must accomplish a homeostatic steady state with their milieu. A paradigmatic example of the cell- microenviromental interplay is gut micro milieu whose integrity is essential for preserving not only the specific tissue homeostasis but also to maintain an efficient mucosal immunity, thus playing a crucial role in healthy status and in various immunological and nonimmunological disorders. A growing number of studies in recent years have highlighted the importance of nutrition and epigenetic modulators as a crucial determinant of health and disease. This review focuses on microRNAs and immune system components in physiological (Fig.1A) and pathophysiological (Fig.1B) gut micro milieu.

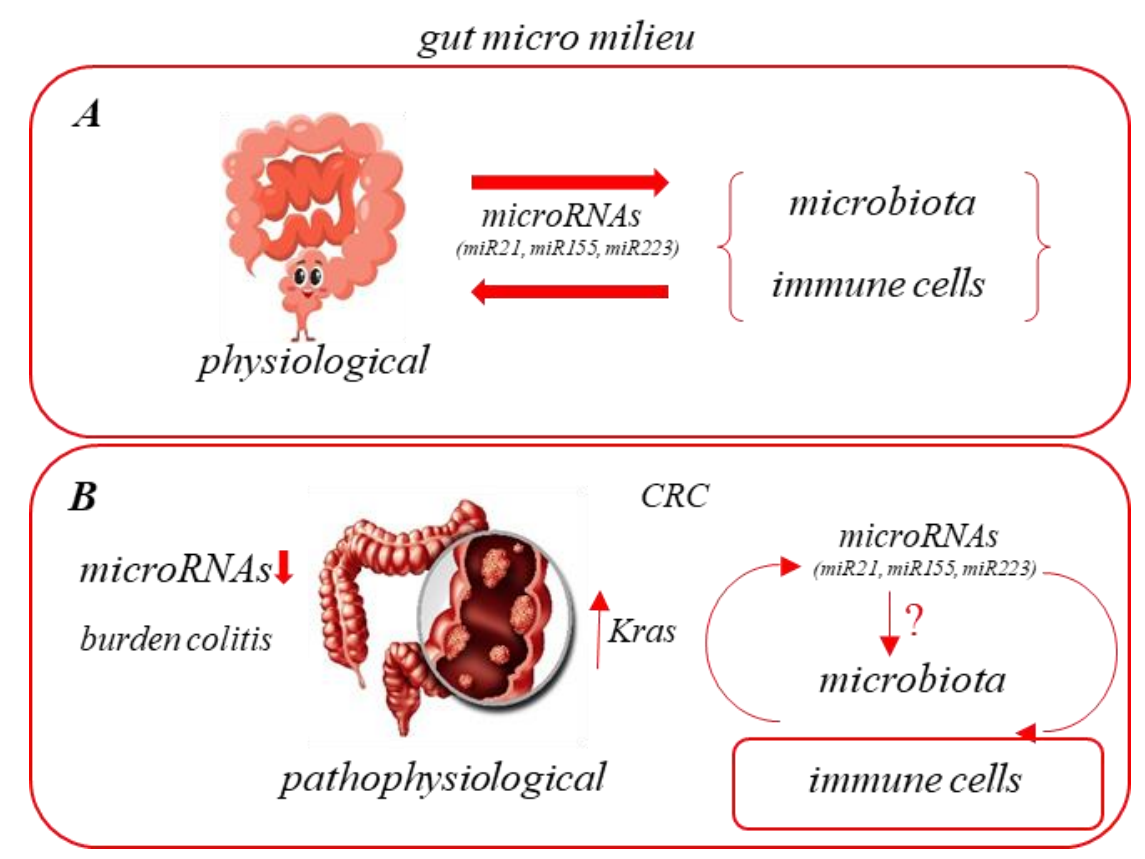

Figure 1: A) miRNAs homeostasis ensure equilibrium of immune cells and microbiota in gut micro milieu. B) Impaired miRNAs expression affect both microbiota and immune cells in colitis. Kras in CRC drive microRNAs cargo release. 


\section{MicroRNAs in physiological gut micro milieu}

Over the last years we gain profound insight in the regulation of immune system and its dialogue with the microbiota in the gut micro milieu. MicroRNAs (miRNAs) released form IECs directly affects bacterial gene expression [6]. In the modern medicine the discovery of miRNAs is undoubtedly a promising field of research for understand more of the complex mechanisms which sustain human health status and to identify new biomarkers for assessing screening test especially for those disease where early hallmarks are essential. MiRNAs are transcribed, as pri-miRNAs, then processed by Drosha [7] into pre-miRNAs and exported into the cytoplasm by Exportin-5 here are further processed by Dicer forming 18 to 22 nucleotides long miRNAs duplexes which is incorporated into ribonucleoprotein complex, known as RISC [8] bring them to modulate translation via complementary base pairing on messenger RNAs (mRNAs). Essential cellular processes such as differentiation, apoptosis and metabolic activities are controlled by specific miRNAs levels and several authors, have demonstrated their essential role of miRNAs in orchestrating immune system logic and its response [9]. IECs show different expression profile of microRNAs between ileum and colon. MiRNAs released in the gut micro milieu can directly affect bacterial gene expression [6]. Gut micro milieu microRNAs come from, not only by EICs now recognized as non-hematopoietic cells exhibiting immunological functions but also by hematopoietic cell populations. Several miRNAs are found in the gut micro milieu. Among them evidenced in multiple studies on human (blood and gut biopsies) the miR-21, miR-155, and miR-223 are indicative of gut disease [10]. Of this triade, the miR-155 has a central regulatory role in macrophages and dendritic cells maturation and is required for gut mucosa protection. In fact, miR$155 \%$ deficient mice has burden colitis. Of interest that miR-155 regulates gut B cells in a similar manner of its action in B cells found in other microenvironments [11]. In addition, high concentration miRNAs including the miR-155, were found in gut luminal content respect to IECs suggesting that these miRNAs are specifically secreted into the gut micro milieu [6]. An important observation on miR-21 and miR-155 is that both, promote microsatellite instability in inflammatory bowel diseases which results from impaired DNA mismatch repair and may induce Kras mutations in colonic mucosa [12; 13]. Of interested that, locked 
nucleic acid (LNA)-modified oligonucleotides (also known as antagomirs) targeting the miR-155 could mitigates immune response in allogeneic bone marrow stem cell transplantation [14].

\section{MicroRNAs in cancer associated gut micro milieu}

It is now accepted that tumor micro milieu is an active player in cancer progression and deserves to be investigated for searching therapeutic targets. Cancer progression is complexly linked to immune response. Activation of specific immune cells such as DCs, NK cells and CD8 ${ }^{+} \mathrm{T}$ cells drive and determine potent anticancer responses. Instead, illogically other components of immune system suppress its anti-cancer responses leading to bypass its immune surveillance and promoting cancer survival. This happen because cancer cells directly suppress specific subsets of immune cells through a myriad of aberrant biochemical mechanisms including the dysregulation of miRNA expression via the release of exosoma-derived microRNAs in the tumor micro milieu. Besides that, cancer cells have been shown to alter their own miRNAs program in order to avoid immune detection. Cancer cells and immune cells can create a "loop" mediated by exosomemiRNAs [15; 16]. It has been proven that exosomal contents modify tumor micro milieu facilitating cell cancer cytoskeleton reorganization, angiogenesis, and influencing tumor immunity. It has been demonstrated that chemicals inducing gastroenteric cancer regulate exosomal miR-155 release [17; 18] but tumor micro milieu and its component is still a dilemma for colorectal cancer [19]. Although hypoxia, typical in CRC is able per se to induce pro-inflammatory cytokines which lead to the upregulation of several microRNAs in both, primary and immortalized CRC [20; 21] and CRC-specific miRNAs can also be detected in stool patients [21] nothing is known about fecal occult blood and miRNAs as well as CRC miRNAs release in the gut micro milieu associated to this type of cancer.

\section{Conclusion}

Essential cellular processes such as differentiation, apoptosis and metabolic activities in gut micro milieu are controlled by specific miRNAs levels. The identification of miRNAs and their role has mRNAs controller added another layer to the complexity of control of gut micro milieu homeostasis. These epigenetic modulators work as a double edge sword. While in physiological condition they promote immune response in gut microenvironment in CRC exosomal microRNAs modify tumor micro milieu favoring cell cancer 
cytoskeleton reorganization, angiogenesis, and influencing metastatic process. Understanding the role of miRNAs in the complex interactions between the gut disease (even tumor), cells and might microbiota in gut microenvironment might provide important information on new potential targets for therapy diagnosis and prognosis in CRC.

\section{Acknowledgements}

We apologize to those whose work was not cited due to time and space constraints. The authors are gratefully to Ministero dell'Universita e della Ricerca (MIUR) (Rome) for “Dipartimento di Eccellenza grant 2018-2022".

\section{Conflict of interest}

The authors declare that they have no conflicts of interests.

\section{References}

[1] M.J. Bissell, H.G. Hall, G. Parry, How does the extracellular matrix direct gene expression? Journal of theoretical biology 99 (1982) 31-68.

[2] A.W. Stoker, C.H. Streuli, M. Martins-Green, M.J. Bissell, Designer microenvironments for the analysis of cell and tissue function. Current opinion in cell biology 2 (1990) 864-874.

[3] C.Q. Lin, M.J. Bissell, Multi-faceted regulation of cell differentiation by extracellular matrix. FASEB journal : official publication of the Federation of American Societies for Experimental Biology 7 (1993) 737-743.

[4] N. Boudreau, C. Myers, M.J. Bissell, From laminin to lamin: regulation of tissue-specific gene expression by the ECM. Trends in cell biology 5 (1995) 1-4.

[5] S. Liu, A.P. da Cunha, R.M. Rezende, R. Cialic, Z. Wei, L. Bry, L.E. Comstock, R. Gandhi, H.L. Weiner, The Host Shapes the Gut Microbiota via Fecal MicroRNA. Cell host \& microbe 19 (2016) 32-43.

[6] A.M. Denli, B.B. Tops, R.H. Plasterk, R.F. Ketting, G.J. Hannon, Processing of primary microRNAs by the Microprocessor complex. Nature 432 (2004) 231-235.

[7] R.I. Gregory, T.P. Chendrimada, N. Cooch, R. Shiekhattar, Human RISC couples microRNA biogenesis and posttranscriptional gene silencing. Cell 123 (2005) 631-640.

[8] A.Mehta, D. Baltimore, MicroRNAs as regulatory elements in immune system logic. Nature reviews. Immunology 16 (2016) 279-294.

[9] J. Lee, E.J. Park, H. Kiyono, MicroRNA-orchestrated pathophysiologic control in gut homeostasis and inflammation. BMB reports 49 (2016) 263-269.

[10]M.C. Runtsch, J.L. Round, R.M. O'Connell, MicroRNAs and the regulation of intestinal homeostasis. Frontiers in genetics 5 (2014) 347.

[11]M. Svrcek, N. El-Murr, K. Wanherdrick, S. Dumont, L. Beaugerie, J. Cosnes, J.F. Colombel, E. Tiret, J.F. Flejou, T. Lesuffleur, A. Duval, Overexpression of microRNAs-155 and 21 targeting mismatch repair proteins in inflammatory bowel diseases. Carcinogenesis 34 (2013) 828-834.

[12] M.H. Lyda, A. Noffsinger, J. Belli, C.M. Fenoglio-Preiser, Microsatellite instability and K-ras mutations in patients with ulcerative colitis. Human pathology 31 (2000) 665-671.

[13] https://clinicaltrials.gov/ct2/show/NCT01521039.

[14] F.J. Kohlhapp, A.K. Mitra, E. Lengyel, M.E. Peter, MicroRNAs as mediators and communicators between cancer cells and the tumor microenvironment. Oncogene 34 (2015) 5857-5868. 
[15] E. Bell, M.A. Taylor, Functional Roles for Exosomal MicroRNAs in the Tumour Microenvironment. Computational and structural biotechnology journal 15 (2017) 8-13.

[16]J. Zhou, X.L. Li, Z.R. Chen, W.J. Chng, Tumor-derived exosomes in colorectal cancer progression and their clinical applications. Oncotarget 8 (2017) 100781-100790.

[17]C. Chen, F. Luo, X. Liu, L. Lu, H. Xu, Q. Yang, J. Xue, L. Shi, J. Li, A. Zhang, Q. Liu, NF-kB-regulated exosomal miR-155 promotes the inflammation associated with arsenite carcinogenesis. Cancer letters 388 (2017) 21-33.

[18] T. Colangelo, G. Polcaro, L. Muccillo, G. D'Agostino, V. Rosato, P. Ziccardi, A. Lupo, G. Mazzoccoli, L. Sabatino, V. Colantuoni, Friend or foe? The tumour microenvironment dilemma in colorectal cancer. Biochimica et biophysica acta 1867 (2017) 1-18.

[19]Z. Shen, R. Zhou, C. Liu, Y. Wang, W. Zhan, Z. Shao, J. Liu, F. Zhang, L. Xu, X. Zhou, L. Qi, F. Bo, Y. Ding, L. Zhao, MicroRNA-105 is involved in TNF-alpha-related tumor microenvironment enhanced colorectal cancer progression. Cell death \& disease 8 (2017) 3213.

[20] A.M. Strubberg, B.B. Madison, MicroRNAs in the etiology of colorectal cancer: pathways and clinical implications. Disease models \& mechanisms 10 (2017) 197-214.

[21]T. Masuda, N. Hayashi, Y. Kuroda, S. Ito, H. Eguchi, K. Mimori, MicroRNAs as Biomarkers in Colorectal Cancer. Cancers 9 (2017). 